\title{
Telling the story of life in the cosmos: the LUVOIR telescope concepts
}

\section{Aki Roberge, Matthew Bolcar, Kevin France}

Aki Roberge, Matthew R. Bolcar, Kevin C. France, "Telling the story of life in the cosmos: the LUVOIR telescope concepts," Proc. SPIE 11115, UV/Optical/ IR Space Telescopes and Instruments: Innovative Technologies and Concepts IX, 1111500 (9 September 2019); doi: 10.1117/12.2530475

SPIE Event: SPIE Optical Engineering + Applications, 2019, San Diego, California, United States 


\title{
Telling the story of life in the cosmos: The LUVOIR telescope concepts
}

\author{
Aki Roberge ${ }^{* a}$, Matthew R. Bolcar ${ }^{\mathrm{a}}$, Kevin C. France $^{\mathrm{b}}$ \\ On behalf of the LUVOIR Study Team \\ ${ }^{a}$ NASA Goddard Space Flight Center, Greenbelt, MD, USA 20771 \\ ${ }^{b}$ Department of Astrophysical and Planetary Sciences, Laboratory for Atmospheric and Space \\ Physics, University of Colorado, Boulder CO, USA 80309
}

\begin{abstract}
The Large UV/Optical/Infrared (LUVOIR) Surveyor is a concept for a powerful general-purpose observatory spanning the far-UV to the near-infrared. Two variants are being studied: LUVOIR-A (15-m diameter primary mirror) and LUVOIR-B (8-m mirror). These powerful and flexible observatories will enable revolutionary new studies in astrophysics and planetary science.

LUVOIR is being designed to take the next great leap in exoplanet studies, with direct images and spectra of rocky Earthsized exoplanets in the habitable zones of other stars. These data will allow a wide range of investigations, including analysis of terrestrial planet atmospheres, discovery of potentially habitable exoplanets, and searches for evidence of global biospheres. A key goal for LUVOIR is to conduct these studies on a set of candidate habitable exoplanets large enough to constrain the frequency of habitable conditions (dozens of rocky planets orbiting solar-type stars).

LUVOIR will also provide revolutionary advances in a broad range of astrophysics - from the epoch of reionization, through galaxy formation and evolution, to star and planet formation. The observatory could also enable powerful remote sensing observations of Solar System bodies. The LUVOIR-A architecture offers up to $25 \mathrm{~km}$ resolution at Jupiter, enabling sensitive, high resolution observations over long time baselines and a broad wavelength range. Finally, perhaps LUVOIR's most important scientific capability is its ability to address not only the science questions of today, but those of the 2040s and beyond that we have not yet thought to ask.
\end{abstract}

Keywords: space telescopes, exoplanets, astrophysics, ultraviolet, optical, near-infrared

\section{INTRODUCTION}

Humanity is defined by the drive to know about the world around us. The value of that quest for the betterment of our species is immeasurable. Ages-old questions and investigations earned us the revelations that the stars are Suns swirling in a vast galaxy, itself one of a myriad of islands in an expanding cosmos. Now we have crossed another threshold of discovery: there are planets around other stars (e.g., Mayor \& Queloz 1995). At this key point in human history, tracing a path from the dawn of the universe to life-bearing worlds is within our grasp.

This monumental objective demands powerful and flexible new tools, as well as application of multi-disciplinary scientific skills. The abundance and diversity of worlds is far greater than imagined, yet there are planetary systems reminiscent of the solar system as well (e.g., Winn \& Fabrycky 2015). At the moment, the vast majority of known exoplanets are "small black shadows" indirectly detected through their effect on their host stars. Our knowledge of exoplanet properties is largely limited to orbits, masses, and sizes. Astronomers have just begun to measure the atmospheric constituents of gas giant exoplanets; such studies will greatly expand in the coming years.

The next frontier is to extend these capabilities to rocky planets and find the "pale blue dots" in the solar neighborhood. With a large enough sample size, scientists can determine whether habitable, Earth-like conditions are rare or common on nearby worlds and then probe them for signs of life (e.g., atmospheric oxygen). Focusing on the planetary systems most like the solar system, those with Earth-size exoplanets orbiting in the habitable zones of Sun-like stars, increases the chances of finding and recognizing atmospheric biosignatures. Concurrently, we will nurture a new discipline -

*Aki.Roberge@nasa.gov; phone 1301 286-2967; https://asd.gsfc.nasa.gov/Aki.Roberge/home.html

UV/Optical/IR Space Telescopes and Instruments: Innovative Technologies and Concepts IX, edited by Allison A. Barto, James B. Breckinridge, H. Philip Stahl, Proc. of SPIE Vol. 11115, 1111500 · (c) 2019 SPIE CCC code: $0277-786 X / 19 / \$ 21 \cdot$ doi: $10.1117 / 12.2530475$ 

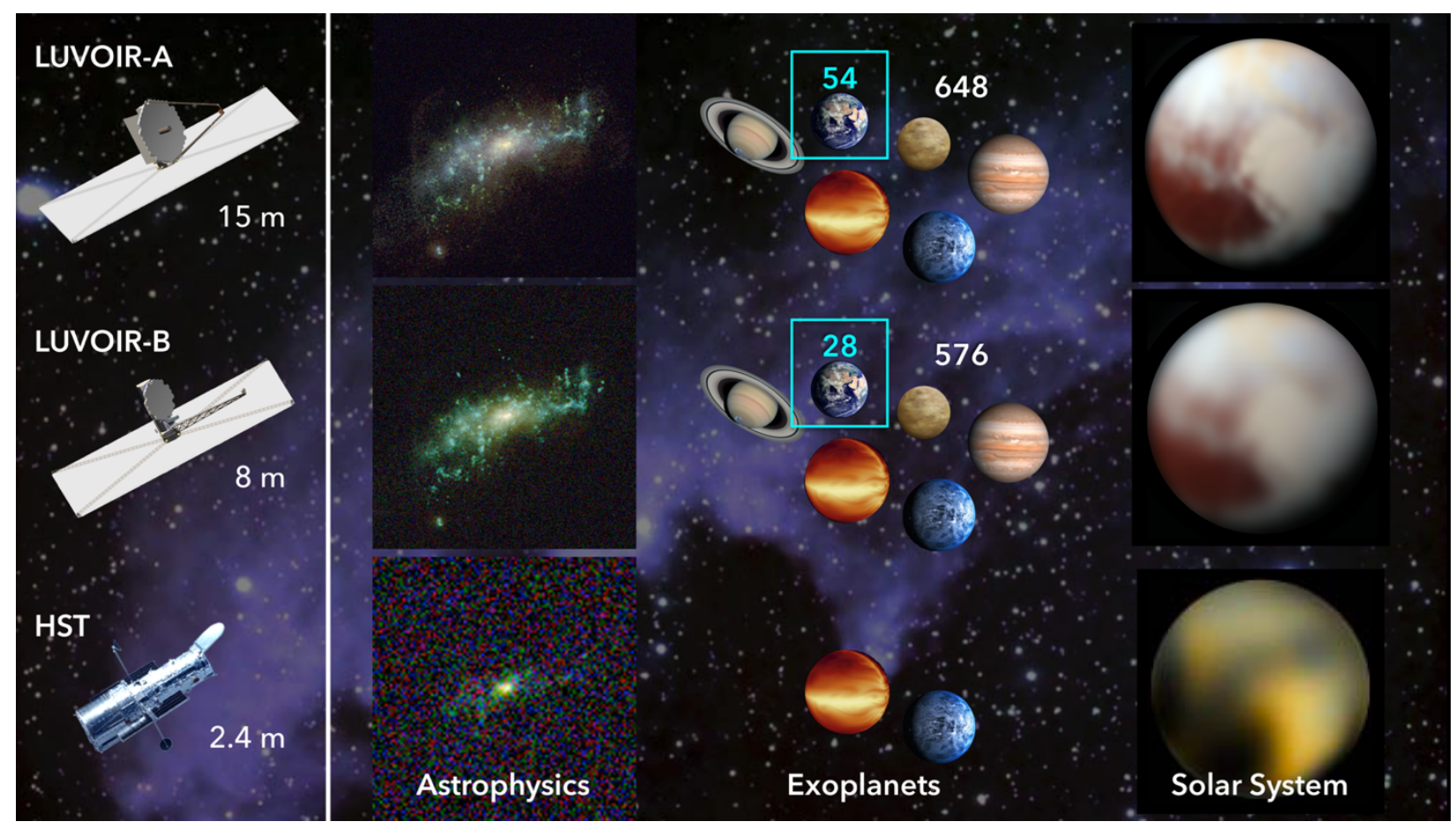

Figure 1: LUVOIR will revolutionize huge areas of space science. Its sensitivity and spatial resolution open the door to the ultra-faint and ultra-distant regime, enabling detailed observations of the full variety of galaxies. LUVOIR dramatically increases the sample size and diversity of exoplanets that can be studied, providing dozens of Earth-like exoplanet candidates that can be probed for signs of life (54 with LUVOIR-A and 28 with LUVOIR-B) and hundreds of non-habitable exoplanets (648 with LUVOIR-A and 576 with LUVOIR-B). Finally, LUVOIR will provide near-flyby quality observations of solar system bodies (HST Pluto image from Buie et al. 2010). Credits: NASA / New Horizons / M. Postman (STScI) / A. Roberge (NASA GSFC)

comparative exoplanetology - by studying a huge range of exoplanets, thereby gaining invaluable information for placing our own system in the broader context of planetary systems. A vital part of establishing that context is deeper understanding of the bodies within the solar system.

Our drive to know goes beyond asking the question "what exists?" to "why does it exist?" and pushes us to understand the origins of all we see around us. The boundary of what we can see now stretches all the way to the dawn of the universe, but like our first steps in the study of new worlds, our vision lacks both completeness and context. We seek to understand the environments and processes that gave rise to a life-supporting cosmos: from the formation of the earliest structures to the assembly and evolution of galaxies, to the detailed mechanisms of star and planet formation. The boundaries of physics will be tested and stretched while exploring the birth and evolution of the cosmos.

These scientific questions and investigations demand an observatory beyond any in existence or in development. They require a large aperture to capture the very faintest objects across cosmic time. They require the ability to block the blinding light from hundreds of stars and observe dozens of small, faint planets orbiting them. They require access to a range of wavelengths broad enough to read the fingerprints of matter across all temperatures and densities. These needs define the required tool, which is the Large Ultraviolet / Optical / Infrared Surveyor (LUVOIR). LUVOIR is a vision for a community facility with the power and flexibility to answer scientists' questions across the full portfolio of Exoplanet, Cosmic Origins, Physics of the Cosmos, and Solar System Exploration science (Figure 1).

The LUVOIR mission concept is one of four Large Mission Concepts studied in preparation for the 2020 Astrophysics Decadal Survey (Astro2020). The study was initiated in Jan 2016, under the leadership of a Science and Technology Definition Team (STDT) drawn from the community (team list at https://asd.gsfc.nasa.gov/luvoir/team/). Over the last 3.5 years, the STDT and the LUVOIR engineering team - with valuable input and assistance from the broader community have worked closely to produce one of the most detailed and mature large mission concepts ever presented to an 
Table 1: LUVOIR's Signature Science Cases

\begin{tabular}{|l|}
\hline \# 1 - Finding habitable planet candidates \\
\hline \# 2 - Searching for biosignatures and confirming habitability \\
\hline \# 3 - The search for life in the solar system \\
\hline \# 4 - Comparative atmospheres \\
\hline \# 5 - The formation of planetary systems \\
\hline \# 6 - Small bodies in the solar system \\
\hline \# 7 - Connecting the smallest scales across cosmic time \\
\hline \# 8 - Constraining dark matter using high precision astrometry \\
\hline \# 9 - Tracing ionizing light over cosmic time \\
\hline \# 10 - The cycles of galactic matter \\
\hline \# 11 - The multiscale assembly of galaxies \\
\hline \# 12 - Stars as the engines of galactic feedback \\
\hline
\end{tabular}

Astrophysics Decadal Survey. The records and results of this work will be submitted to NASA HQ and Astro2020 in the form of a Final Report and additional technical material (e.g., master equipment lists). The Final Report is available at https://asd.gsfc.nasa.gov/luvoir/.

The scope of science enabled by LUVOIR is truly vast, encompassing all the topics addressed by the Hubble Space Telescope (HST) and more. The LUVOIR STDT therefore decided to focus on a set of "Signature Science Cases" (Table 1). In Chapters 3-6 of the LUVOIR Final Report, we explain the motivations for the Signature Science Cases, identify key measurements, and set out needed observations. The telescope and instrument characteristics required for the observations are also identified. We have developed concrete observing programs for each Signature Science Case to ensure that the LUVOIR designs can execute this compelling science within the 5-year prime mission lifetime (Appendix B of the Final Report).

The Signature Science Cases represent some of the most compelling observing programs that scientists might do with LUVOIR at the limits of its performance. As compelling as they are, they should not be taken as a complete specification of LUVOIR's future scientific potential. Additional science cases contributed by the STDT and the broader community appear in Appendix A of the Final Report. We fully expect that the creativity of the community, empowered by the revolutionary capabilities of LUVOIR, will ask questions, acquire data, and solve problems beyond those discussed in the Final Report. Just as HST is today doing science not envisioned at the time of its design or launch, LUVOIR's power, flexibility, and potential longevity will allow it to execute the as-of-yet unknown science of the 2040s and beyond.

\section{OVERVIEW OF THE LUVOIR MISSION CONCEPTS}

The LUVOIR Team developed two distinct observatory concepts: the 15-m LUVOIR-A (Figure 2), designed for launch in NASA's Space Launch System (SLS) Block 2 vehicle; and the 8-m LUVOIR-B (Figure 3), designed to fit in heavy-lift launch vehicles with 5-m fairings similar to those in use today. By studying two designs, we gain better understanding of a complex trade space, reveal how science return scales with different technical choices, and establish robustness to uncertainties such as future launch vehicle capabilities and budget constraints. It is important to recognize that LUVOIRA and -B represent proof-of-concept point designs within a family of UV/Optical/NIR observatories, demonstrating feasibility and providing information for the future. More complete information on the LUVOIR designs and technology appears in a companion SPIE proceeding [1]. LUVOIR's main features are: 


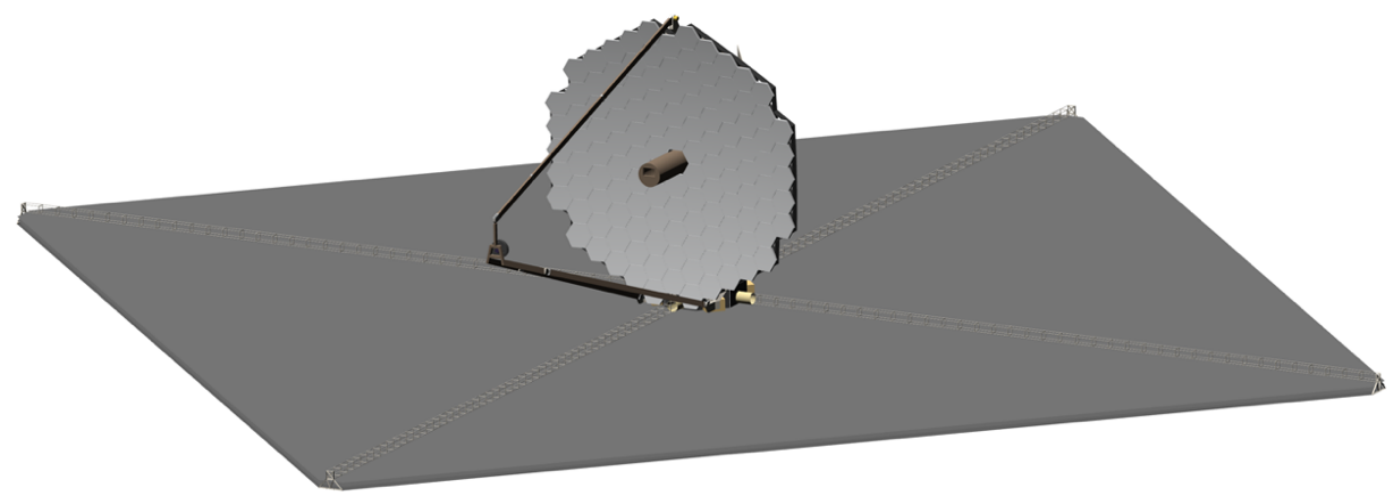

Figure 2: The LUVOIR-A observatory, with a 15-m diameter on-axis primary mirror and four instruments bays. Deployment and pointing animation at https://asd.gsfc.nasa.gov/luvoir/design/. Credit: A. Jones (NASA GSFC)

- Scalable architecture for a servicable observatory at Sun-Earth L2

- A 5-year prime mission, with 10 years of on-board consumables. Non-servicable components have a 25-year lifetime goal

- Segmented, deployable telescopes designed for launch in next-generation heavy lift vehicles with large fairings (e.g., NASA's SLS Block 2, NASA's SLS Block 1B Cargo, Blue Origin's New Glenn, and SpaceX's Starship)

- UV-capable telescopes compatible with high-contrast exoplanet observations (total wavelength range of $100-2500 \mathrm{~nm}$ )

- Telescope optical designs to alleviate polarization effects that can degrade coronagraph contrast

- A sunshade that is larger but simpler than the James Webb Space Telescope (JWST) sunshield (three layers instead of JWST's five, relaxed requirements on layer positioning after deployment)

- A field-of-regard that covers most of the sky. LUVOIR can point to within $45^{\circ}$ of the Sun, greatly enhancing target of opportunity, exoplanet, and solar system observations

- Moving target capability with tracking speed of $60 \mathrm{mas} / \mathrm{sec}$ (2x faster than JWST)

- Extreme Coronagraph for LIving Planetary Systems (ECLIPS): A high-contrast coronagraph with imaging cameras and integral field spectrographs spanning 200-2000 nm, capable of directly observing a wide range of exoplanets

- High Definition Imager (HDI): A high spatial resolution camera covering 200-2500 nm, incorporating high precision astrometry capability

- LUVOIR Ultraviolet Multi-Object Spectrograph (LUMOS): A far-UV imager and multi-resolution, multi-object spectrograph covering 100-1000 nm, capable of simultaneous observations of up to hundreds of sources

- POLLUX: A high-resolution, point-source UV spectropolarimeter covering 100-400 nm, designed for LUVOIRA. This instrument study was contributed by a consortium of European institutions, with leadership and support from the French Space Agency

A transformative facility like LUVOIR with its large aperture and need for high-contrast observations of Earth-like exoplanets requires new approaches to designing and realizing an observatory. Far more than previous space telescopes, LUVOIR must be considered as an integrated system and was designed for adaptability:

- Scaleable architecture to leverage a rapidly changing launch vehicle landscape

- Modular design to ease on-orbit servicing and integration \& testing on the ground

- Several layers of active wavefront control on the telescopes and within instruments to relax constraints on hardware precision and on-the-ground verification 


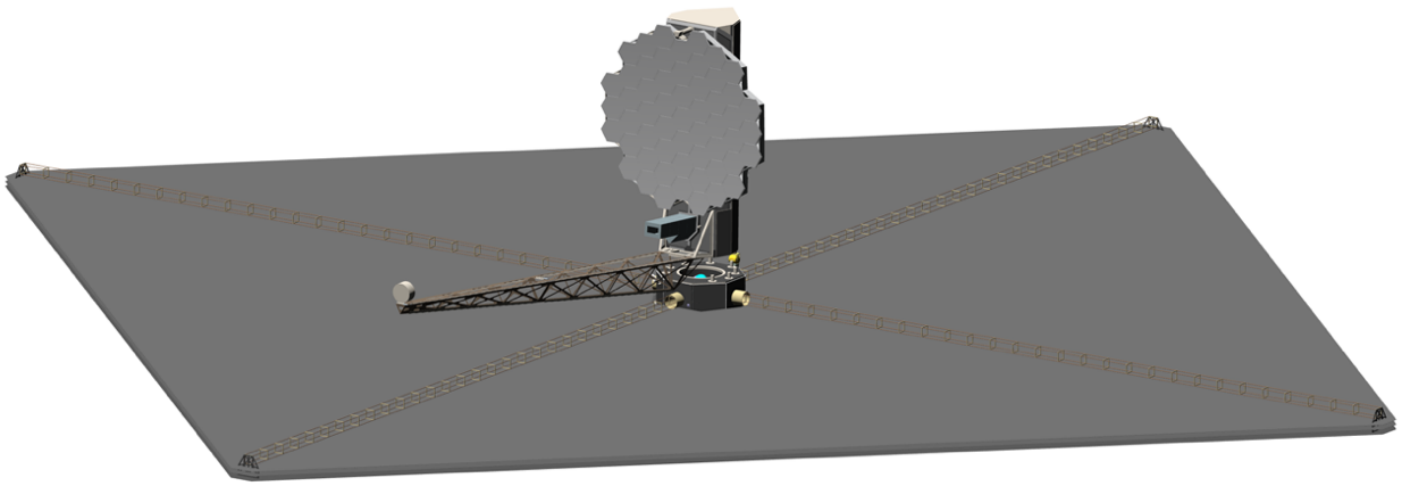

Figure 3: The LUVOIR-B observatory, with an 8-m diameter off-axis primary mirror and three instruments bays. Deployment and pointing animation at https://asd.gsfc.nasa.gov/luvoir/design/. Credit: A. Jones (NASA GSFC)

- Minimization and isolation of vibrations and thermal disturbances throughout the observatory

\section{KEY SCIENCE GOALS AND OBJECTIVES}

\subsection{Signature Science Case \#1: Finding habitable planet candidates}

Indirect planet discovery techniques have shown that small, rocky planets are not rare. Results from NASA's Kepler mission indicate that the fraction of Sun-like stars with roughly Earth-size planets in their habitable zones, is about $24 \%$ [2]. These zones span the range of distances from the stars where we think rocky planets can have liquid water - an essential material for all life on Earth - on their surfaces. This revelation drives us to proceed to the next step: build the capabilities to reveal the character of rocky exoplanets and measure the fraction that are truly Earth-like (a.k.a. exoEarths). We need to probe rocky planet atmospheres and determine their thermal/chemical states by measuring molecular abundances, including water vapor and other greenhouse gases.

Furthermore, measuring the frequency of habitable conditions requires observations of a large number of candidate exoplanets (dozens). To find and study that many candidates, an even larger number of stars must be observed (hundreds). A systematic survey of exoplanets most similar to the Earth - rocky planets in the habitable zones of Sun-like stars guarantees that whatever we find will be of scientific value. In the absence of water vapor detections, we would know that global-scale surface oceans are rare on rocky worlds in the habitable zone. This null result would still transform our understanding of habitability as a planetary process, and further confirm the special nature of our home. On the other hand, if we find that Earth-like conditions are common on rocky exoplanets, then a stunning vista of hospitable new worlds will be unveiled.

Scientists have calculated that to study the habitability of rocky planets around Sun-like stars, we must collect and analyze light from the planets themselves, i.e. obtain direct images and spectra [3]. However, direct observations of rocky exoplanets around Sun-like stars demand the very high contrast possible only with a space telescope coupled to a highperformance starlight suppression system [3]. The LUVOIR Final Report describes the survey strategy developed to obtain the needed measurements while dealing with all of the expected complexities in an efficient manner, and the calculations to determine expected exoplanet yields. The expected exoplanet yields obtained in initial 2-year coronagraph surveys with LUVOIR-A and -B returned by our analysis appears in Figure 4. The LUVOIR-B observatory meets the requirement set by the LUVOIR STDT for a statistically significant exoEarth candidate survey (28 habitable planet candidates). With 54 habitable planet candidates, LUVOIR-A surpasses the requirement and provides ample margin against astrophysical and technological uncertainties. The same code with the same astrophysical input assumptions was used to calculate exoplanet yields for the Habitable Exoplanet Observatory (HabEx) mission concept.

Figure 5 shows that the expected yield of habitable planet candidates is a strong function of telescope diameter and changes with aperture geometry; these realities drove the design of the LUVOIR concepts. The inscribed diameter is the diameter of the largest circle completely contained within the telescope primary aperture; this is the parameter that has the single greatest impact on yields. The yields also depend on whether the aperture is unobscured (off-axis) or obscured (on-axis), which affects the choice of coronagraph type. In Figure 5, off-axis monolithic and segmented telescopes are shown with 


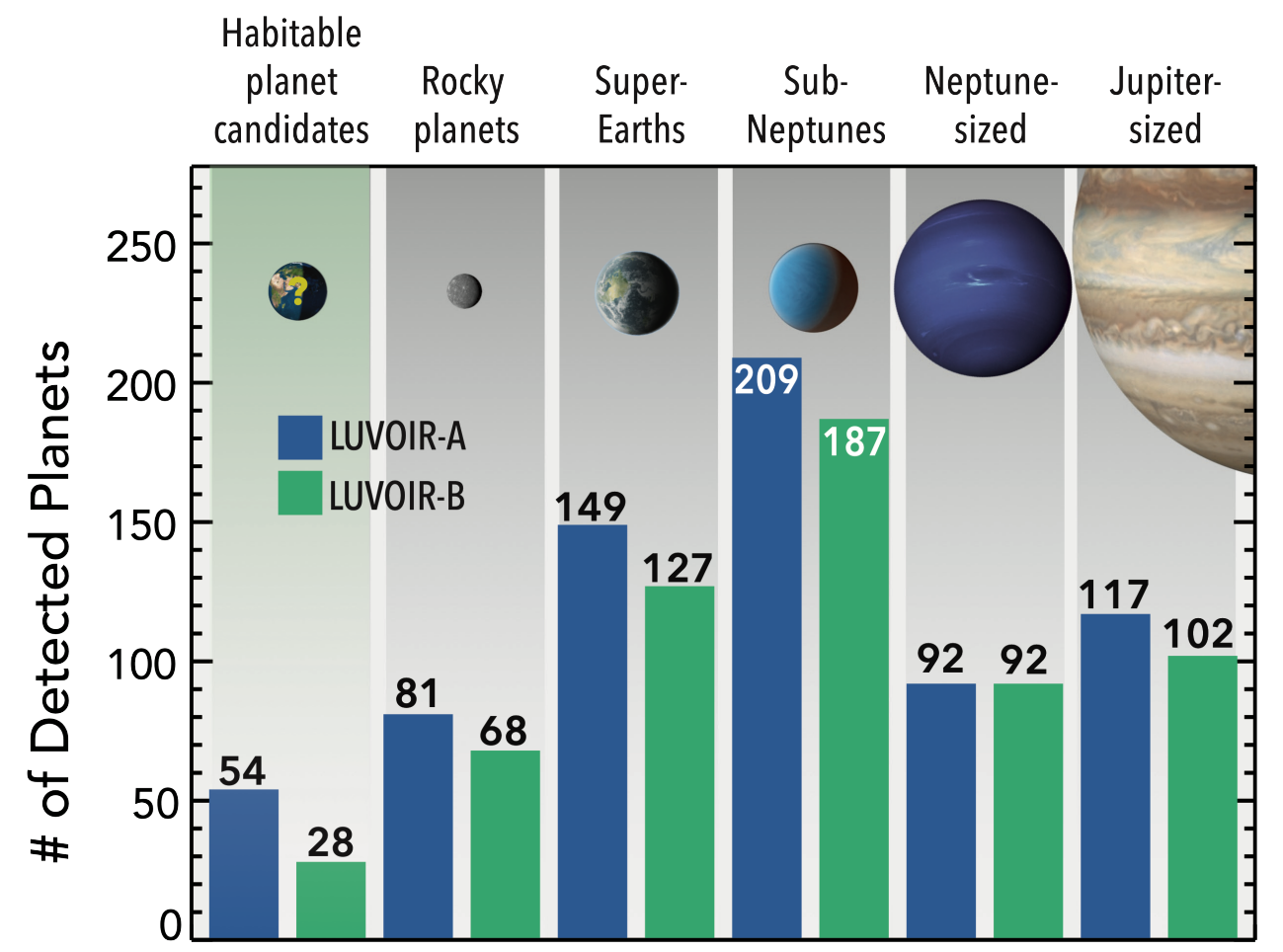

Figure 4: LUVOIR discovers dozens of habitable planet candidates and hundreds of other kinds of exoplanets. The chart shows exoplanet detection yields from an initial 2-year survey optimized for habitable planet candidates with LUVOIR-A (blue bars) and -B (green bars). The first column shows the expected yields of habitable planet candidates. Non-habitable planets are detected concurrently during the 2-year survey. Color photometry is obtained for all planets. Orbits and partial spectra capable of detecting water vapor and/or methane are obtained for all habitable planet candidates. Credit: C. Stark (STScI) / J. Friedlander (NASA GSFC)

the green and red curves, respectively. Both sets of curves end at the largest size that appears feasible for that type of telescope. The range between the upper and lower curves spans different approaches to observing strategy. The off-axis telescopes are paired with vector vortex charge 6 (VC6) coronagraphs. The smooth joining of the green and red curves show that new coronagraph designs have eliminated any penalty for segmentation.

On-axis segmented telescopes are shown with blue curves in Figure 5. They are paired with apodized pupil Lyot coronagraphs (APLC), which perform better with obscured apertures than VC6. The yield gap between red and blue curves shows that there remains a penalty for obscuration. The two black points show the expected yields for LUVOIR-B $(6.7-\mathrm{m}$ inscribed diameter, off-axis segmented paired with VC6) and LUVOIR-A (13.5-m inscribed diameter, on-axis segmented paired with an APLC). The yellow curve shows the expected yield from a single starshade paired with a 4-m telescope and without refueling of the shade's propulsion system. This curve demonstrates that, unlike coronagraph-only systems, the yield from starshade-only systems does not continue increasing with telescope diameter.

\subsection{Signature Science Case \#2: Searching for biosignatures and confirming habitability}

LUVOIR will not only achieve the exoEarth census but will probe the atmospheres of those planets for biosignature gases, possibly revealing the first signs of life outside our home planet. The spectrum of the Earth contains features arising from gases of biological origin, like $\mathrm{O}_{2}$ and methane, in addition to features from water vapor and other greenhouse gases (Figure 6). The Earth is the only planet we know of teeming with surface life that strongly affects the planet's atmosphere. Thus, there are good reasons to focus serious searches for life outside the solar system on exoplanets that are as much like the Earth as possible. By targeting exoplanets with sizes and orbits similar to Earth's and host stars similar to the Sun, we hope to increase our chances of finding - and recognizing - extraterrestrial life.

However, it is important to understand that no one molecule (or pair of molecules) is automatically a biosignature in every context. Many studies over the last decade have identified pathways to atmospheres containing abundant oxygen without 


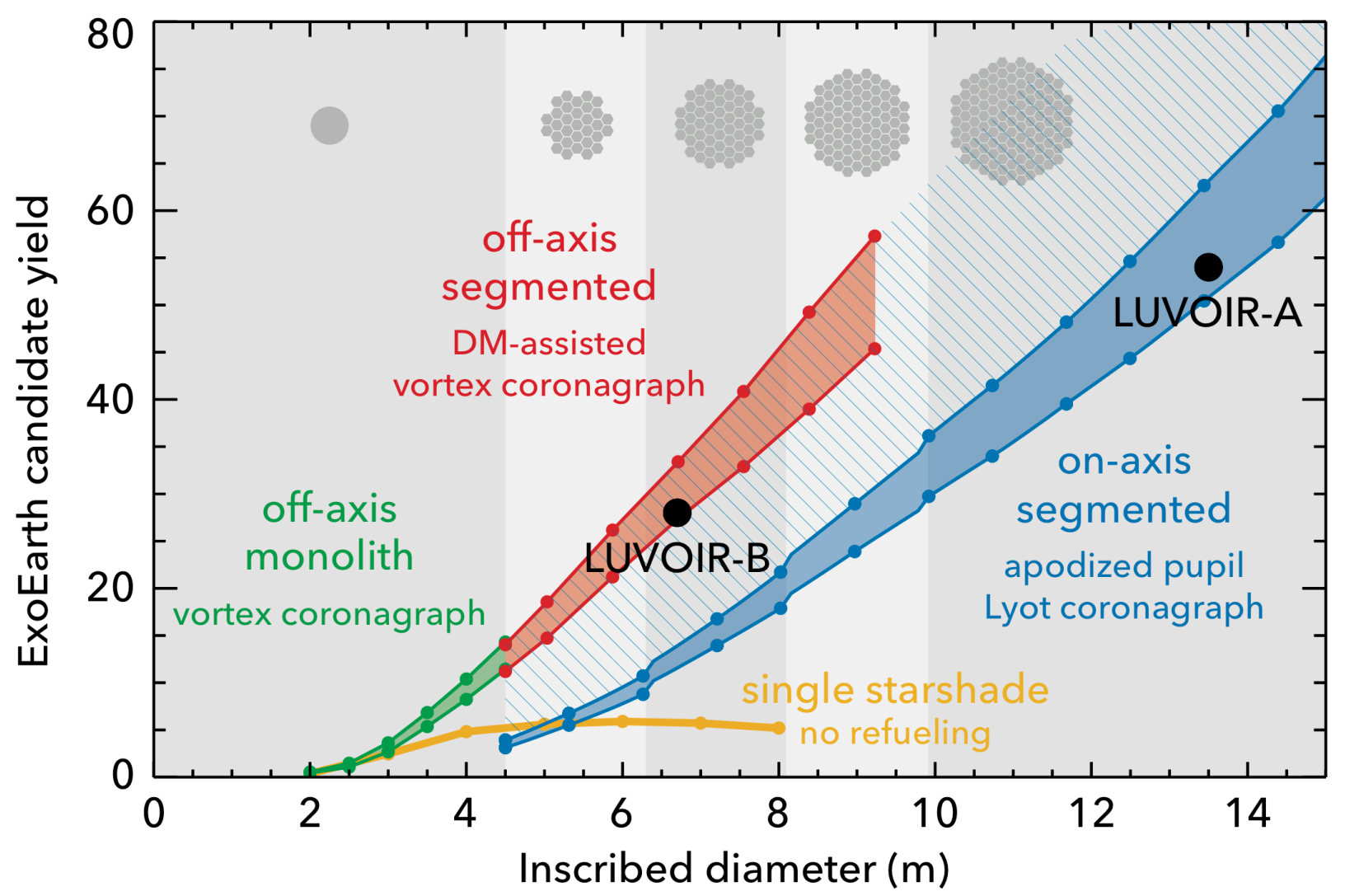

Figure 5: Telescope size, aperture geometry, and coronagraph type all affect the expected detection yields of exoEarth candidates. On the $\mathrm{x}$-axis, the inscribed diameter is the diameter of the largest circle completely contained within the telescope aperture. The green, red, and blue curves show yields for different combinations of telescope aperture geometry and coronagraph type, more fully explained in the main text. The yellow curve shows the yields for a single starshade paired with a 4-m telescope. Credit: Stark et al. (2019)

life, especially for planets around $\mathrm{M}$ dwarf stars [4]. The key is to measure the abundances of many molecules in the whole atmosphere, and then use those abundances to estimate the production and destruction rates predicted by physics and chemistry. To calculate the production and destruction rates, a good deal of knowledge about the atmosphere's context will be needed, including the planet's orbit, mass, and the characteristics of the host star. Scientists will try to explain the character of an atmosphere with two sets of models: one where the physics and chemistry of the atmosphere are purely driven by geological and astrophysical processes; and a second where biological sources are also considered. Those planets for which only the second set of models can explain the data are those for which evidence of life may be robustly claimed.

Therefore, confident life-detection has at least three fundamental requirements: 1) assess a wide range of atmospheric molecules, 2) measure or constrain their abundances with some precision, and 3) understand the planet's context within its system. The first requirement demands direct spectra with broad wavelength coverage from the near-ultraviolet (NUV) to the near-infrared (NIR). The LUVOIR telescopes and starlight suppression systems can span these wavelengths of light and access the important constituents of planetary atmospheres, including water $\left(\mathrm{H}_{2} \mathrm{O}\right)$, carbon dioxide $\left(\mathrm{CO}_{2}\right)$, molecular oxygen $\left(\mathrm{O}_{2}\right)$, ozone $\left(\mathrm{O}_{s}\right)$, and methane $\left(\mathrm{CH}_{4}\right)$. Important indicators of false positive biosignatures (e.g., $\left.\mathrm{CO}\right)$ can be observed as well. The second requirement demands good quality spectra to measure abundances, which drives the required telescope and instrument sensitivity. The third requirement demands collection of a range of supporting information, including planet masses and far-UV spectra of stellar activity-driven emission from the host stars. LUVOIR's great sensitivity and flexibility will enable the variety of observations needed to properly understand a planet as a complete system.

\subsection{Signature Science Case \#3: The search for habitable worlds in the solar system}

The search for habitable environments also takes place closer to home. Scientists have discovered that several moons of the outer solar system have liquid water beneath their icy surfaces. These sub-surface oceans must be heated from below, 

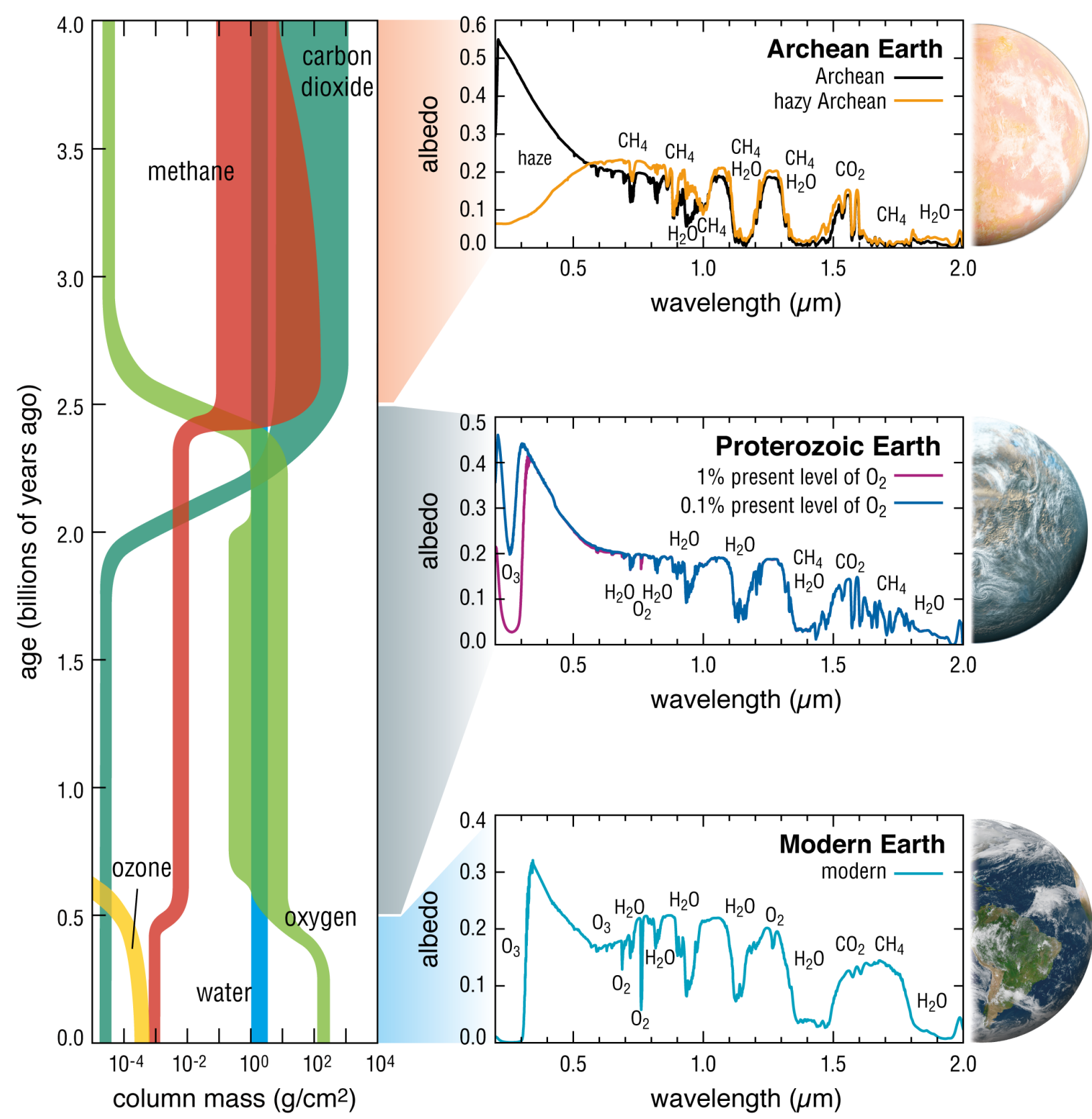

Figure 6: Earth's atmospheric composition has changed significantly over its inhabited history (left), as has its spectral features (right). For instance, the canonical biosignatures of modern Earth (e.g. $\mathrm{O}_{2}, \mathrm{O}_{3}$ ) cannot be observed in the Archean eon. A wide wavelength range enables higher fidelity spectral characterization by providing observations of many atmospheric species, and often multiple absorption bands of a given species. This breaks degeneracies between overlapping bands (e.g., overlapping $\mathrm{CH}_{4}$ and $\mathrm{H}_{2} \mathrm{O}$ absorption features in the near-infrared) and enables the search for non-traditional biosignatures. Credit: G. Arney, S. Domagal-Goldman, T. B. Griswold (NASA GSFC)

which may also provide the energy needed for life. Plumes emanating from openings in the icy shells, such as those observed from Europa and Enceladus [5,6], may allow glimpses into the deep oceans. LUVOIR has an important role to play in determining the currently unknown strength and frequency of plume activity by providing observations over long time baselines. Such information will be a valuable support for future spacecraft visiting these other ocean worlds to search for signs of life with focused investigations. The FUV multi-object spectroscopy capability of LUMOS is particularly well suited to spectroscopic imaging of auroral emission from Europa's plumes in multiple atomic lines (e.g., the neutral 


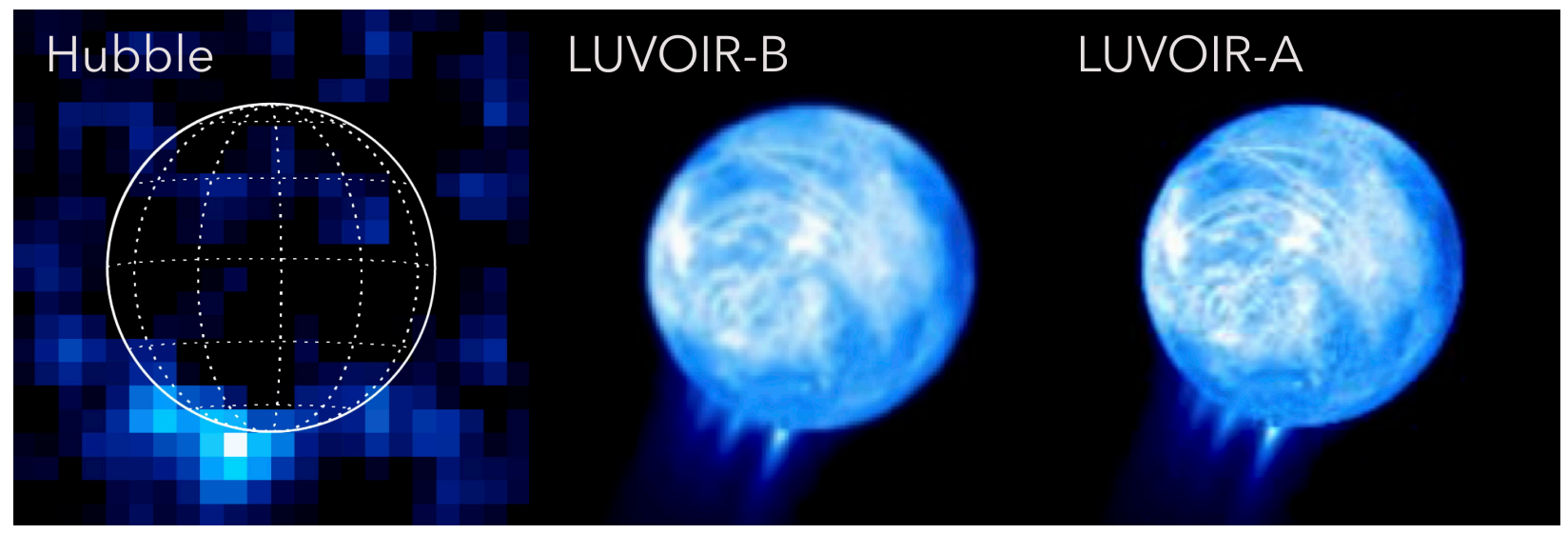

Figure 7: LUVOIR can monitor individual plumes from solar system ocean moons. The left panel shows an aurora on Europa observed with HST [5]. This far-UV hydrogen emission comes from dissociation of water vapor in plumes escaping through the moon's ice shell. The center and right panels show simulations of how this emission might look observed with LUMOS on LUVOIR-B and -A. The moon's surface is bright due to reflected solar hydrogen emission, which was below the background in the HST image. Credit: G. Ballester (LPL) / R. Juanola-Parramon (NASA GSFC)

hydrogen Lyman $\alpha$ line at $121.5 \mathrm{~nm}$ and the neutral oxygen line at $130.4 \mathrm{~nm}$ ). Furthermore, the large apertures of the LUVOIR telescopes may permit emission from individual jets to be spatially resolved (Figure 7). This key feature will aid disentangling plume strength vs. plume morphology.

\subsection{Signature Science Case \#4: Comparative atmospheres}

The capabilities that will allow LUVOIR to investigate dozens of potentially habitable worlds will also allow comprehensive study of a huge range of exotic exoplanets, which by and large will be easier to observe than Earth-like exoplanets. Many of these comparative planetology studies cannot be done by studying the planets of the solar system alone; for example, investigations of the effects of host star mass, stellar metallicity, system age, and a broad range of planet masses and orbits. For this diverse set of planets, the primary objectives are to measure the abundances of the primary atmospheric constituents; characterize hazes and clouds; and assess atmospheric loss rates. These objectives will be achieved with a combination of direct and transit spectroscopy, both obtained with LUVOIR. The initial 2-year survey for habitable planet candidates will uncover large numbers of other types of exoplanets (Figure 4), which will range from hot rocky planets to cold giant planets.

\subsection{Signature Science Case \#5: The formation of planetary systems}

LUVOIR will provide unprecedented measurements to help understand the processes that give rise to the extraordinary diversity of planetary systems found over the last decades. LUVOIR will examine the formation and evolution of planetary systems over a wide range of stellar ages by executing unprecedented spectroscopic studies of the most abundant materials in protoplanetary disks (Figure 8); revealing the morphology of young planetary systems; and providing the data for dynamical studies of mature planetary systems to uncover clues to their formation eons ago.

\subsection{Signature Science Case \#6: Small bodies in the solar system}

There are many things still to be discovered and understood about the bodies within the solar system itself. Sensitive, high resolution imaging and spectroscopy of solar system asteroids, dwarf planets, comets, and Trans-Neptunian Objects (TNOs) that will not be visited by spacecraft in the foreseeable future can provide vital information on the processes that formed the solar system ages ago. In particular, the Kuiper Belt is the only available remnant of the solar system's primordial planetesimal population accessible for direct study. The characteristics of this population, including both size and orbital distributions, are directly relevant to understanding process of planetesimal formation and the migration of the giant planets. In particular, the size distribution of the smallest bodies provides critical constraints on theories of the formation of the first planetary building blocks.

HST remains the most sensitive optical telescope ever built, with the ability to detect TNOs with diameters in the range of $\sim 35 \mathrm{~km}$ at 40 AU. However, this limit may leave $99 \%$ of the inner TNO population invisible to observers. Deep imaging programs with LUVOIR can detect smaller TNOs than any other current or planned observatory: $\sim 2 \mathrm{~km}$ bodies at $40 \mathrm{AU}$ 

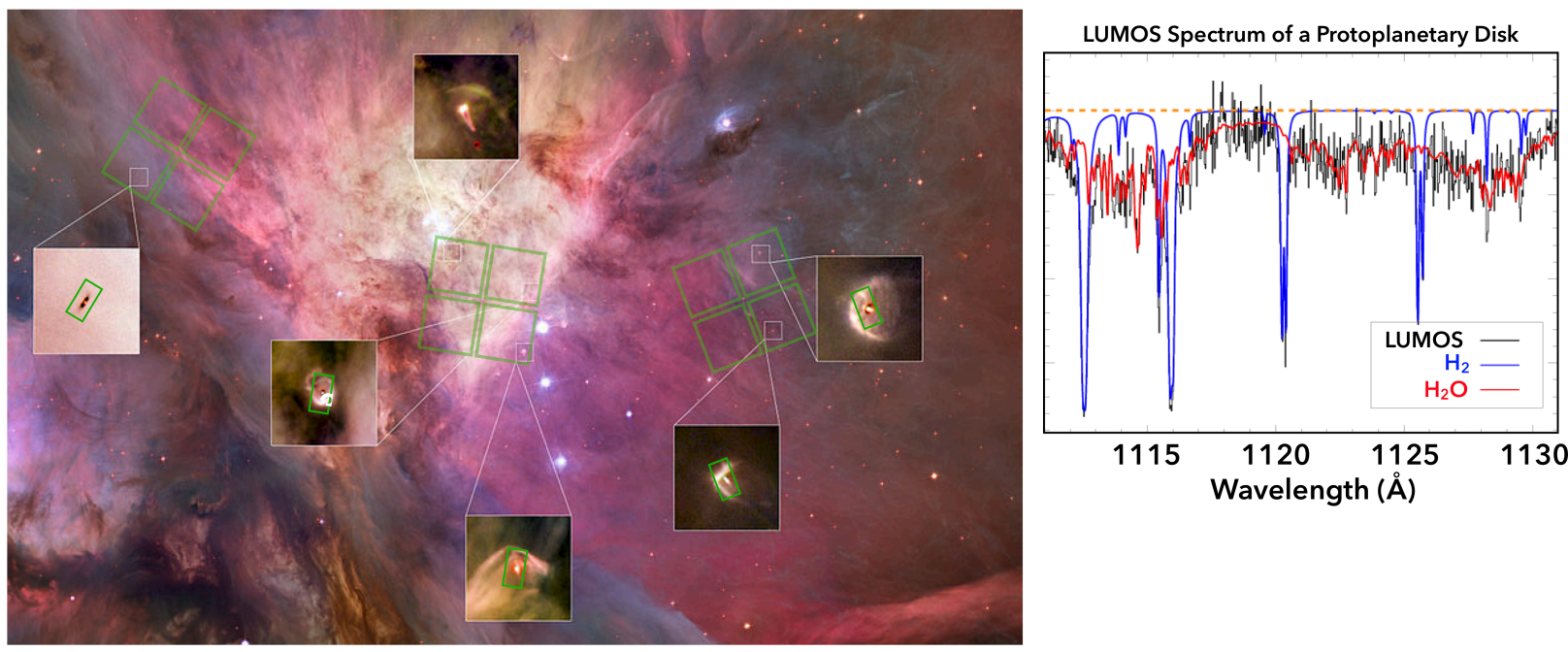

Figure 8: LUVOIR can powerfully and efficiently measure the hidden materials of planet formation. Left: The Orion Star Forming Region with the LUMOS multi-object spectroscopic field of view overlaid three times (green squares). The blow-up panels show several planet-forming disks that fall in each field, with LUMOS spectroscopic apertures overlaid (green rectangles). UV spectra of all the disks in each field can be obtained simultaneously. Right: Simulated partial LUMOS spectrum of a protoplanetary disk showing absorption from two key molecular species $\left(\mathrm{H}_{2}\right.$ and $\left.\mathrm{H}_{2} \mathrm{O}\right)$. Credit: NASA / ESA / HST / K. France (CU - Boulder) / J. Tumlinson (STScI)

with LUVOIR-A and $\sim 4 \mathrm{~km}$ with LUVOIR-B. These size limits are two orders of magnitude smaller than the limit for the Large Synoptic Survey Telescope (LSST) wide field survey and an order of magnitude smaller than the limits for proposed or potential deep drills with JWST and future ground-based 30-m-class telescopes (Extremely Large Telescopes, or ELTs).

\subsection{Signature Science Case \#7: Connecting the smallest scales across cosmic time}

The Universe began smoothly, but is now richly structured on all scales. The prevailing cold dark matter (CDM) paradigm for structure formation describes how the first seeds of structure evolved into the dynamic shape of the "cosmic web" of filaments and voids traced out by galaxies. The most current CDM models accurately predict the numbers and properties of "dark matter halos" where galaxies and clusters of galaxies form. However, fundamental questions remain unsolved: What is the dark matter? Do dark matter-based models of structure formation correctly predict small (sub-galactic) size scales? Does dark matter decay? CDM models were built to explain large-scale structure. Whether they can explain smaller structures is a key test that will reveal whether we have captured the physics of interactions between normal matter, dark matter, and ionizing light.

Dwarf galaxies are highly sensitive to the nature and distribution of dark matter - such as the particle mass and any selfinteractions - because almost all their mass is dark matter. The space density and internal structure of dwarf galaxies are closely connected to the fundamental properties of the dark matter particle, the history of reionization, and the granular limits of the galaxy formation process. Each type of dark matter property manifests itself on astronomical scales through a gravitational signature, in the statistical description of dark matter structure. The measure of this signature is the matter power spectrum today and at earlier times. LUVOIR will measure the matter power spectrum by mapping the regions around spiral galaxies out to $\sim 15 \mathrm{Mpc}$ to find low-mass dwarf galaxies, far more sensitively than JWST and the ELTs.

\subsection{Signature Science Case \#8: Constraining dark matter using high precision astrometry}

A complementary constraint on the nature of dark matter comes from using the orbits of stars within dwarf spheroidal galaxies (dSphs) to study their density profiles. These galaxies are dark matter-dominated, and their central density profiles are a sensitive probe of dark matter properties. Those density profiles can be measured using the 3D motions of individual stars within the galaxies. However, for the smallest dSphs that are most dominated by dark matter, current and planned observatories cannot precisely measure transverse velocities for sufficient numbers of stars to accurately determine the galaxies' central density profiles. LUVOIR can provide the necessary velocity precision for enough stars, thanks to its angular resolution, sensitivity, and a pixel geometry calibration system within the HDI instrument. LUVOIR will measure the proper motions of stars within dSphs with $\sim 10 \mathrm{~km} / \mathrm{s}$ accuracy out to $4 \mathrm{Mpc}$ with LUVOIR-A or $2 \mathrm{Mpc}$ with LUVOIR- 


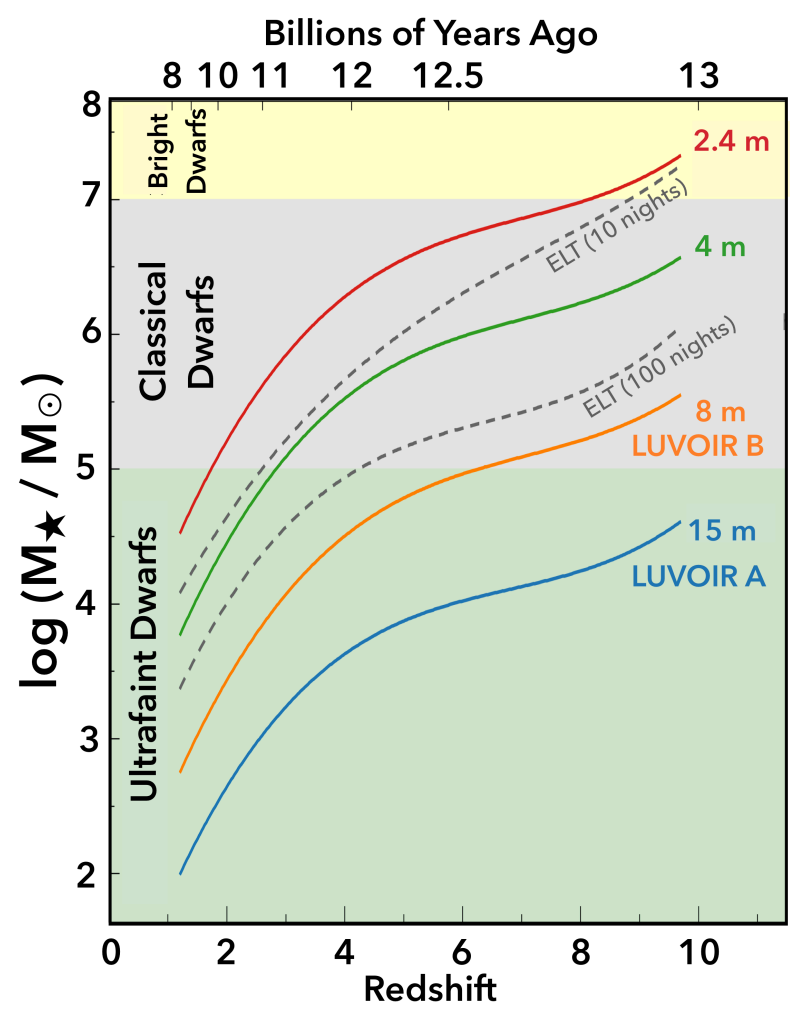

Figure 9: LUVOIR can detect smaller galaxies than any other current or planned observatory. The curves show sensitivity to detecting galaxies of a particular total stellar mass (y-axes) as a function of redshift (and lookback time) for different telescopes: a 2.4-m space telescope, a 4-m space telescope, the LUVOIR concepts, and the 39-m ground-based ELT. The limits for the space-based telescopes are for a fiducial $500 \mathrm{ksec}(139$ hour) observation that returns a SNR=5 detection of a 200-parsec diameter source. The limits for the ELT are for a 10-night (80 hour) integration and a 100-night (800 hour) integration. Results for JWST are similar to those for the ELT 100-night exposure. Credit: M. Postman (STScI)

B. Combined with radial velocities from the ELTs, these proper motion measurements will provide galaxy density profiles and dark matter maps in cases where it is not currently possible due to the difficulty of obtaining transverse velocities.

\subsection{Signature Science Case \#9: Tracing ionizing light across cosmic time}

The major open questions of cosmic structure formation go far beyond the nature of dark matter. The opacity and temperature of the intergalactic medium (IGM) influences where and how gas can enter dark matter halos to form stars, making the history of "reionization" a major unsolved problem. The problem is complex because dwarf galaxies are the predominant sources of ionizing photons and are also most greatly impacted by being ionized and heated. LUVOIR will push beyond JWST and the ELTs to constrain the number and distribution of the faintest galaxies at high redshift - those that feel the effects of reionization most directly. Figure 9 shows the redshift to which telescopes of different sizes can detect dwarf galaxies. With deep imaging of $12 \mathrm{HDI}$ fields in the I, J, and $\mathrm{H}$ bands, obtained in parallel with exoplanet observations, LUVOIR-A can measure photometric redshifts for galaxies as faint as $\mathrm{AB} \approx 33 \mathrm{mag}$, corresponding to the mass of the Milky Way's Fornax dwarf spheroidal galaxy at redshift $z=7$. This unprecedented depth - two magnitudes deeper than JWST - will definitively constrain the faint end slope of the galaxy luminosity function, which in turn constrains the critical influence of reionization on early galaxy formation. However, this specific observing program requires unfeasibly long times with LUVOIR-B.

\subsection{Signature Science Case \#10: The cycles of galactic matter}

Galaxies are built from gas that surrounds them and fills up their disks. Gas flows from the space between the galaxies (the intergalactic medium; IGM), into the region around a galaxy (CGM), then into the galaxy itself where it can become the fuel for new stars. Eventually, massive stars, supernovae, and AGN drive material back out. Much of this cycling of matter is currently unobserved, since the gas flows are hot and tenuous. LUVOIR's uniquely powerful multi-object UV 
spectroscopy will reach an entirely new discovery space in the exploration of galactic gas flows. LUMOS has an array of 840 x 420 individually configurable microshutters, each of which covers 0.07 " x 0.14 ". This angular resolution corresponds to $<100 \mathrm{pc}$ out to $\mathrm{z}=0.1(400 \mathrm{Mpc})$ and $<1 \mathrm{kpc}$ at all redshifts. The ability to probe the powerful UV diagnostics of diffuse gas, hot stars, and dust at such small spatial scales over wide fields will permit the very complex interactions of these processes to be studied as a function of local conditions.

\subsection{Signature Science Case \#11: The multiscale assembly of galaxies}

Since the original Hubble Deep Field in 1996, tracing the growth of galaxies has pushed all the way back to about 400 million years after the Big Bang $(\mathrm{z} \sim 11)$. JWST will press further back and farther down the galaxy mass function with rest-frame optical light observed in the near- and mid-IR. Because of its large size and optimization for optical wavelengths, LUVOIR with HDI will push up to 3 mag fainter. Reaching AB=33-34 mag at the diffraction limit, LUVOIR will exquisitely image the detailed assembly of galaxies from their smallest parts. Telescope aperture is the critical factor enabling this capability, since it sets the spatial resolution and the photometric depth that can be achieved in reasonable times. Figure 9 shows that LUVOIR can detect analogs of the Milky Way's smallest satellite galaxies at the time they were forming stars $(z>4)$. The LUVOIR-A deep fields discussed in Section 3.9 will capture the high-redshift building blocks of larger galaxies, thus achieving another science goal with the same dataset.

Supplementing the LUVOIR deep fields with additional optical images at shorter wavelengths achieves yet another goal: resolving individual star-forming regions within moderate-redshift $(\mathrm{z} \sim 1-2)$ galaxies. These images will have a spatial resolution that Hubble only achieves for the very limited set of gravitationally lensed galaxies (Figure 10). Finally, LUVOIR can reveal the story of star formation in individual galaxies by detecting the main-sequence turnoff in colormagnitude diagrams of resolved stellar populations, providing their ages and metallicities. Previous observing programs could only achieve this for very nearby galaxies, limiting studies to dwarf galaxies and a couple of giant spirals; JWST will be similarly limited. LUVOIR will enable these studies out to the distances needed to reach the nearest giant elliptical galaxies.

\subsection{Signature Science Case \#12: Stars as engines of galactic feedback}

Stars themselves are the most numerous sources of feedback on galaxies, returning energetic radiation to their environments over their lives and substantial amounts of kinetic energy and heavy elements when they die. With its high spatial resolution and uniquely powerful UV spectroscopy, LUVOIR will make fundamental contributions to our understanding of the stellar initial mass function (IMF) in two key areas: very massive stars (VMSs) and stellar multiplicity. VMSs (stars with $\mathrm{M}_{*}>150 \mathrm{M}_{\odot}$ ) can heavily influence their surrounding environment, providing 25-50\% of the ionizing flux from the host cluster. Diagnostics for VMSs lie in the UV, through emission and absorption lines in the 120-200 nm range. Characterizing VMSs and other massive stars will require resolving young and massive star clusters out to $\sim 100 \mathrm{Mpc}$ and obtaining multiplexed UV spectra to measure stellar and wind lines.

Most stars, including massive stars, are in binary and multiple systems. Knowledge of stellar multiplicity and system properties (number of companions, distribution of short vs. long periods, distributions of mass ratios and eccentricities, and their dependence on the stellar mass) provides tight constraints on models of star formation, the IMF, and star cluster evolution. Establishing the impact of local and global conditions on the multiplicity of B type and lower-mass stars will require a census of both short-period and long-period binaries in massive star clusters and in galaxies other than the Milky Way and the Magellanic Clouds. Short-period spectroscopic binaries will be studied with adaptive optics-assisted spectrographs on the ELTs. However, studies of long-period binaries require high angular resolution, a very stable pointspread function, and photometry across entire arcmin fields-of-view, as well as UV multi-object spectroscopy to characterize the stars in the system. LUVOIR will obtain such observations for binary star systems in nearby galaxies.

\subsection{Magnetic fields everywhere: the science of POLLUX}

POLLUX is a high-resolution, point-source UV spectropolarimeter studied and designed by a consortium of European institutions, with leadership and support from the Centre National d'Etudes Spatiales (CNES). The instrument was designed to occupy the fourth instrument bay on LUVOIR-A, and represents a potential future international contribution to the LUVOIR mission. As the POLLUX study is separate from the overall NASA-led LUVOIR study, POLLUX's science goals supplement the main LUVOIR science goals and are discussed along with the instrument's technical details in Chapter 13 of the Final Report. The most innovative characteristic of POLLUX is its spectropolarimetric capability, which enables studies of a broad range of important astrophysical environments that are out of reach for standard highresolution spectroscopy. Spectropolarimetry enables the detection and characterization of magnetic fields and local 


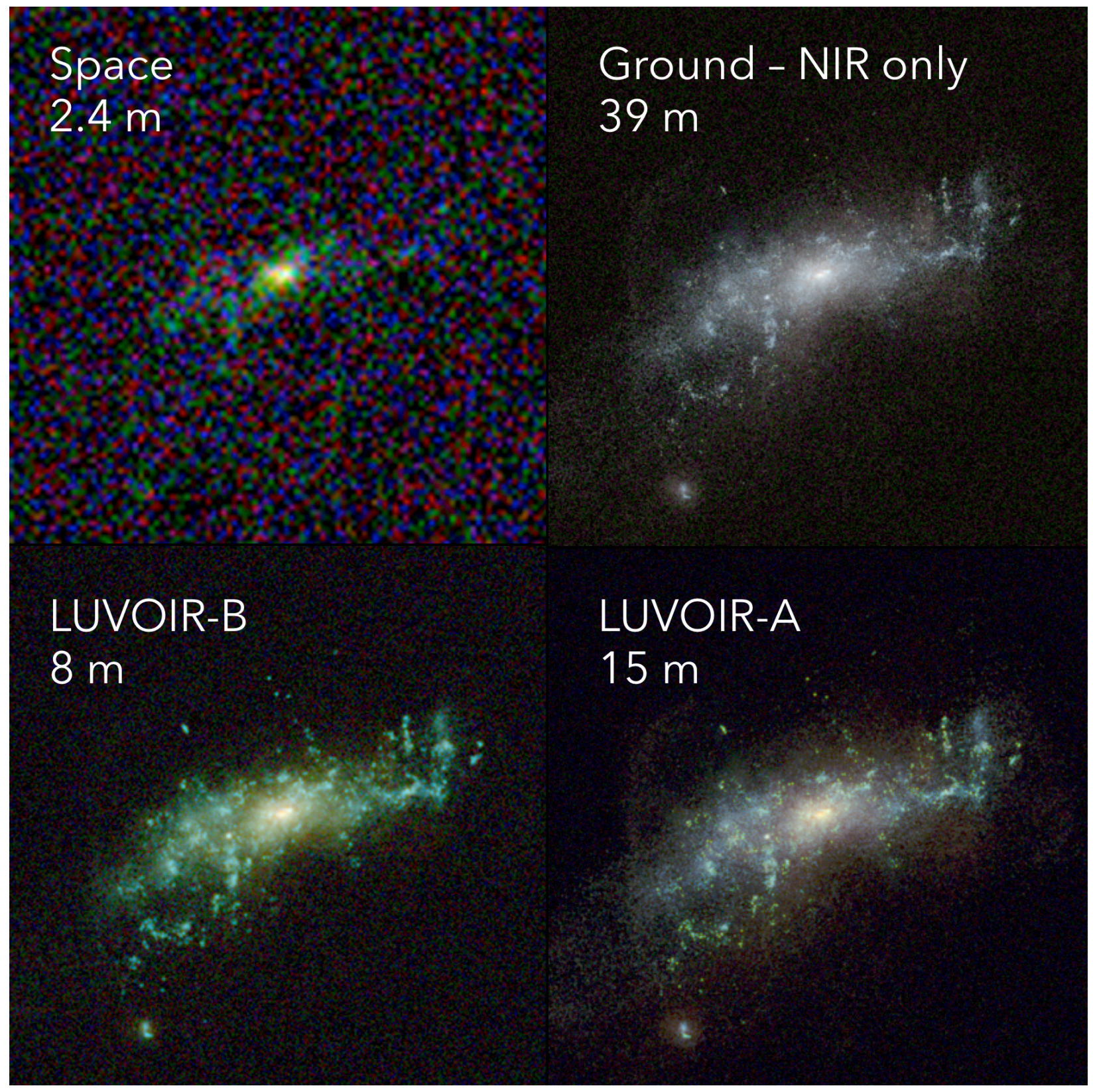

Figure 10: LUVOIR can routinely reveal the internal details of galaxies. Simulations of a low mass $\left(10^{9} M_{\odot}\right)$ galaxy at $\mathrm{z}=2$ imaged with a 2.4-m space telescope (top left), a 39-m ground-based telescope (top right), and LUVOIR (bottom panels). The space-based simulations are a combination of $\mathrm{B}, \mathrm{I}$, and $\mathrm{J}$ band optical images. The ground-based simulation uses longer wavelengths (Y, J, and $\mathrm{H}$ bands), where extreme adaptive optics systems may provide the best spatial resolution. These simulations all assume the same total exposure time (500 ksec, corresponding to 17 nights for the ground-based simulation). Credits: M. Postman, G. Snyder (STScI)

environments of astrophysical objects. POLLUX will address a variety of science cases related to LUVOIR's science portfolio, thereby complementing the other instruments.

\section{LUVOIR'S TOTAL PRIME MISSION SCIENCE}

The Signature Science Cases introduced here and presented in detail in Chapters 3-6 of the LUVOIR Final Report represent a set of programs that push both LUVOIR architectures to their limits, roughly analogous to the Treasury 


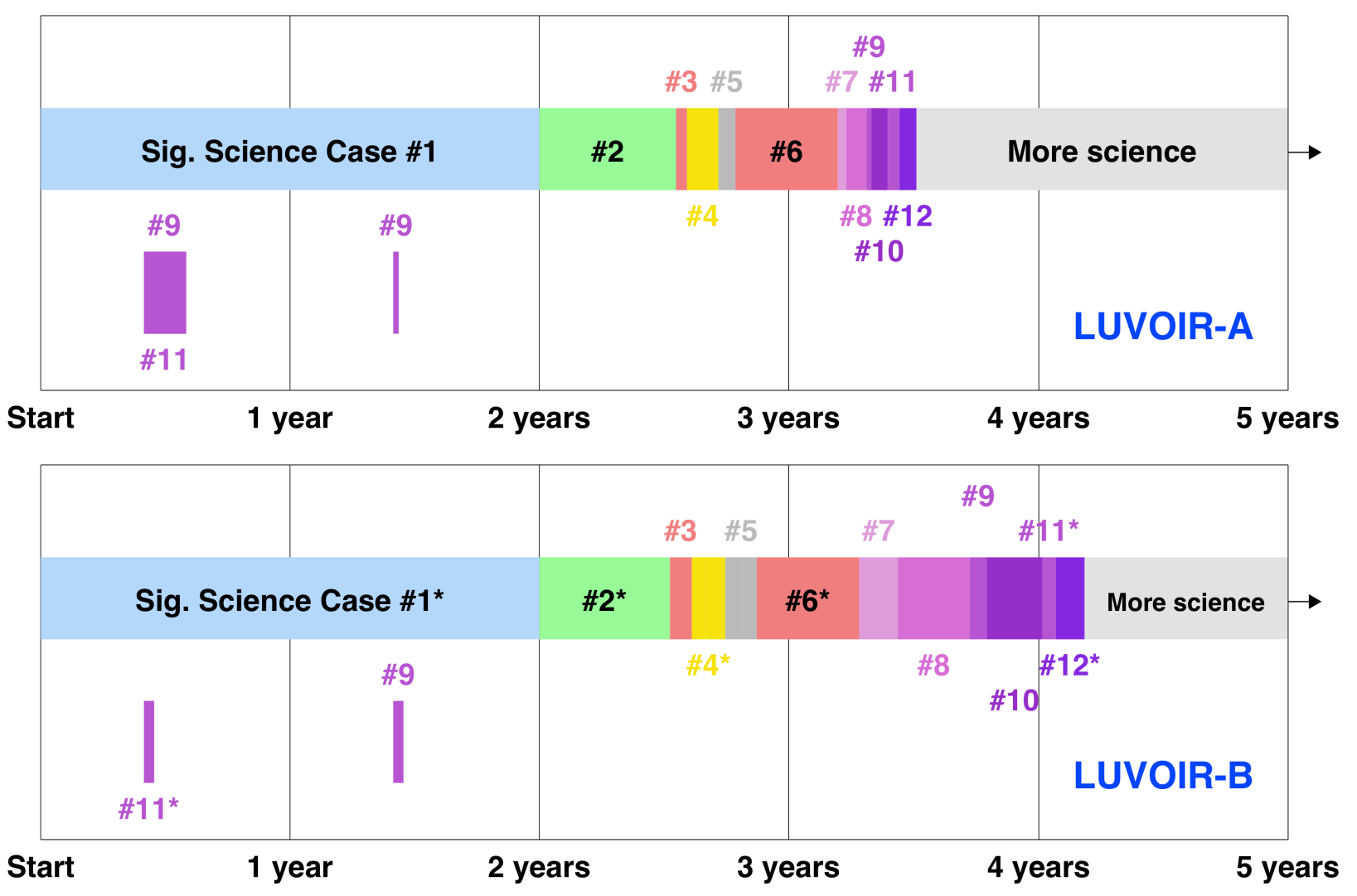

Figure 11: LUVOIR can execute all the Signature Science Case (SSC) observing programs with time to spare in the 5-year prime mission lifetime. Programs that can be executed in parallel with other observations are shown offset below the main bars and do not count against the cumulative time. For LUVOIR-A, one program in SSC \#11 uses the same data as one of the SSC \#9 programs to achieve a different science objective. For LUVOIR-B, an asterisk indicates that the program provides reduced science return (e.g., fewer targets observed). In the most extreme case, the joint SSC \#9/ \#11 dataset mentioned above is deemed unfeasible with LUVOIR-B and therefore omitted. In reality, the observations for these programs will not be scheduled in this order, or all in single continuous time blocks, but will be interleaved. The SSCs take a total of 3.5 years with LUVOIR-A and 4.2 years with LUVOIR-B, leaving time for additional science programs. Credit: A. Roberge (NASA GSFC)

programs on HST today. While the actual science the community chooses to do with LUVOIR can and will change after it launches, it remains important to show that the key science outlined in the Final Report can be performed with the designed hardware in a notional five-year prime mission. Included with the Final Report are scientific Design Reference Mission (DRM) documents that correspond to the Signature Science programs from Chapters 3-6. Target and observation descriptions, as well as exposure times and estimates for overheads, are provided for both LUVOIR-A and B. Figure 11 shows a cumulative representation of these DRMs.

A number of the science cases (e.g., many of the exoplanet-focused programs) are given the same time allocation between LUVOIR-A and B, yielding different quantitative science returns (e.g., number of exoEarth candidates). Others demand the same science return from LUVOIR-A and -B and thus require longer exposure times with LUVOIR-B. Two programs require a deep survey for ultra-faint dwarf galaxies (in Signature Science Cases \#9 and \#11) that was deemed to need an unfeasibly long time with LUVOIR-B. These programs were therefore excluded from the B versions of those Signature Science Cases. Some Signature Science programs can be executed in parallel with ECLIPS exoplanet observations.

The key result from the total DRM analysis is that both LUVOIR-A and LUVOIR-B can perform their ambitious Signature Science Case programs within five years. In fact, both observatories allow significant remaining time for other science programs like those found in Appendix A of the Final Report. The community will choose LUVOIR's science program. But even the most ambitious science programs the LUVOIR Study Team can imagine today, compressed into the first five years of LUVOIR's lifetime, leave significant opportunity for additional transformational science. 


\section{REFERENCES}

[1] Bolcar, M. "The Large UV/Optical/Infrared (LUVOIR) surveyor: engineering design and technology overview," Proc. SPIE, 11115-24 (2019).

[2] Kopparapu, R. K., Hébrard, E., Belikov, R., et al. "Exoplanet Classification and Yield Estimates for Direct Imaging Missions," ApJ, 856, 122 (2018).

[3] National Academies of Sciences. "Exoplanet Science Strategy", Washington, DC: The National Academies Press. https://doi.org/10.17226/25187

[4] Meadows, V. S., Reinhard, C. T., Arney, G. N., et al. "Exoplanet Biosignatures: Understanding Oxygen as a Biosignature in the Context of Its Environment," Astrobiology, 18, 630 (2018).

[5] Roth, L., Saur, J., Retherford, K. D., et al. “Transient Water Vapor at Europa's South Pole,” Science, 343, 171 (2014).

[6] Hansen, C. J., Esposito, L., Stewart, A. I. F., et al. "Enceladus' Water Vapor Plume,” Science, 311, 1422 (2006). 